\title{
A Study of Highly Crosslinked Epoxy Molding Compound and its Interface with Copper Substrate by Molecular Dynamic Simulations
}

\author{
Shaorui Yang, Feng Gao and Jianmin Qu \\ ${ }^{1}$ Department of Civil and Environmental Engineering, Northwestern University \\ 2145 Sheidan Rd, IL, USA 60201 \\ Email: shaoruiyang2009@u.northwestern.edu,feng-gao@northwestern.edu,j-qu@northwesern.edu
}

\begin{abstract}
A novel Epoxy Molding Compound (EMC) with a crosslinked network structure was formed by curing tri-/tetrafunctionalized EPN1180 with Bisphenol-A. A full atomistic model reflecting the network nature of the material was constructed by applying an iterative crosslinking algorithm to an amorphous cell with 3D periodic boundary condition containing the stoichiometric mixture of constitutive monomers. The geometry of the model was then optimized using the COMPASS force-field in Materials Studio [1]. The variation of system density and volume against temperature was simulated using a cooling down profile, which was employed to derive the glass transition temperature and coefficient of thermal expansion of the system. Furthermore, the Young's modulus and Poisson's ratio were calculated by uni-axial tensile molecular statics simulations. The material properties computed by molecular dynamics/mechanics simulations were in good agreement with experiment measurements. An epoxy resin/copper interface model was constructed and the interfacial adhesion energy was calculated as the energy difference between the total energy of the entire system and the sum of the energies of individual materials. The traction-displacement law of the interface was derived when the system was subjected to a molecular statics uniaxial tension. The work of separation and the peak traction, considered as the two key parameters required by cohesive zone finite element simulation, were extracted from the traction-displacement law.
\end{abstract}

\section{Introduction}

Epoxy resin has been widely used in electronic packaging, either in bulk forms such as encapsulants and carriers, or as adhesives, for example die-attach and underfill. The performance of the material itself and its interface with other components, such as metal or metal-oxide substrates, are critical to the reliability of electronic packages. As a matter of fact, the interface between epoxy molding compound (EMC) and copper is one of the weakest links, and has always been a concern in electronic packaging design. To minimize expensive trial-and-error testing, quantitative simulation techniques need to be developed to predict material and interface properties. Despite of the fact that current continuum-based finite element models have become powerful engineering tools, these phenomenological models rely intrinsically on extensive characterization efforts to quantify the parameters present in these models. In addition, the on-going miniaturization down to nano-scale in packaging design imposes a limit to the applicability of continuum models. On the other hand, molecular dynamics and/or mechanics (MD/MM) simulation is able to describe material behavior at atomistic level and thus provide insightful understanding of basic origins of material performances. It is also promising in predicting material properties and establishing constitutive laws, which can be feed to upperscale modeling and simulation such as finite element analysis.

Epoxy resins are thermosetting polymers, having crosslinked network instead of linear or branched chain structure. Due to structural complexity, atomistic simulation of thermosetting polymers has not been carried out until recent years. Several authors have proposed methodologies of building atomistic network structure for thermosetting polymers. Some of these methods [2-10] involve a polymerization molecular dynamics simulation starting from either crystalline [3, 6] or amorphous $[2,4,5,7-10]$ mixtures of separated monomers. Covalent bonds formation between reactive sites followed by relaxation of the system proceeds cyclically, leading to the final crosslinked model with relatively high conversion. Some other authors [11-16], however, followed a simpler approach: they manually designed a segment of a fully cured network instead of letting monomers react to reach whatever configurations they finally arrived at. Various material properties of epoxy resins and other types of thermosets, including glass transition temperature $[2,6,7,12,13,15,16]$, thermal expansion coefficient $[6,7,12]$, mechanical properties $[2,8,12,15,16]$, moisture diffusion behavior $[10,11,14,17]$ and molecular interactions with nano-sized inclusions such as alumina [16] particle and carbon nanotube $[4,5,18]$, have been investigated by $\mathrm{MD} / \mathrm{MM}$ simulations based on molecular models built by both aforementioned methods. Simulation results were in reasonably good agreement with experimental values, indicting the effectiveness of $\mathrm{MD} / \mathrm{MM}$ as a tool to study properties of thermosetting polymers.

Performance of interface between epoxy resins and inorganic substrates has also been investigated by molecular simulation. Interfacial adhesion energy between epoxy resin and copper $[19,20]$ and alumina [10] substrates were calculated as the energy difference between the total energy of the whole system and the sum of the energies of individual materials. Fan et al. [21, 22] studied interfacial separation behavior of copper-epoxy molding compound systems and obtained the traction-separation relationship, serving as the constitutive law of the interface in their cohesive zone finite element simulations. Interfacial separation between inorganic walls and polymers, not necessarily thermosets, has been examined more thoroughly by several other authors [23-25] based on simpler polymer models. Awasthi et al. [24] studied the traction-displacement behavior of graphene-polyethylene interface for both opening and sliding separation mode, and discussed the effects of constraint conditions and size of the model on results. Kulmi and Basu [25] as well as Adnan and 
Sun [23] investigated the competition between adhesive failure along interface and cohesive failure within polymer.

In this study, a fully atomistic model of a novel epoxy molding compound reflecting its infinite network nature was built by an iterative crosslinking algorithm. Material properties, including glass transition temperature, volumetric coefficient of thermal expansion and mechanical behaviors were investigated by molecular dynamics/statics simulation. An epoxy resin/copper interface model was then constructed to study interface adhesion and separation. The constitutive law of the interface, represented as a traction-displacement relationship, was extracted by molecular statics simulation of tensile test

\section{Atomistic simulation methodology and results \\ Molecular structure of the epoxy molding compound}

The epoxy molding compound in this study is composed of tri/tetra-functionality Novolac Epoxy (EPN1180) as resin and bi-functionality Bisphenol-A (BPA) as curing agent. The molecular structures of the two molecules are shown in Fig. 1. The average functionality of EPN1180 is 3.6 ( $\mathrm{q}=1$ or $\mathrm{q}=2$ in Fig. 1 (a)). Fig. 2 describes the curing reaction mechanism through which the epoxy molding compound is synthesized. The epoxy ring on EPN1180 opens in the presence of catalyst. The oxygen atom remains attached to the second epoxy carbon and takes one hydrogen atoms from the hydroxyl group on BPA. Covalent bonds then form between the first epoxy carbons on EPN1180s and the hydroxyl oxygens on BPAs. A schematic illustration of the network structure of the synthesized epoxy molding compound is shown in Fig. 3.

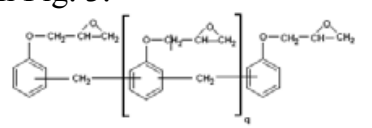

(a)

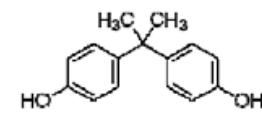

(b)
Figure 1. Molecular structure of (a) EPN1180 and (b) BPA [26]

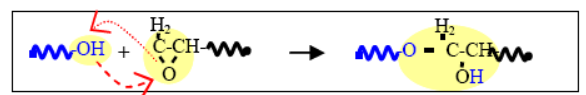

Figure 2. Curing reaction mechanism [26]

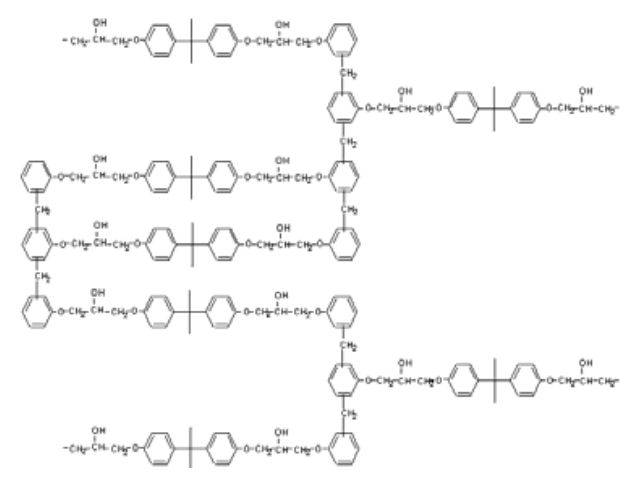

Figure 3. Network structure of the epoxy molding compound [26]

\section{Forcefield selection}

The commercial molecular simulation software Material Studio 4.4 was used for all molecular dynamics/statics simulations in this study. The $a b$ initio Condensed-phase Optimized Molecular Potentials for Atomistic Simulation
Studies (COMPASS) [27] was chosen for the description of intra- and inter-molecular interactions, with the analytical expression as following:

$$
\begin{aligned}
& E_{\text {total }}=\sum_{b}\left[k_{2}\left(b-b_{0}\right)^{2}+k_{3}\left(b-b_{0}\right)^{3}+k_{4}\left(b-b_{0}\right)^{4}\right]+ \\
& \sum_{\theta}\left[H_{2}\left(\theta-\theta_{0}\right)^{2}+H_{3}\left(\theta-\theta_{0}\right)^{3}+H_{4}\left(\theta-\theta_{0}\right)^{4}\right]+ \\
& \sum_{\phi}\left[V_{1}(1-\cos \phi)+V_{2}(1-\cos 2 \phi)+V_{3}(1-\cos 3 \phi)\right]+\sum_{\chi} K_{\chi} \chi^{2}+ \\
& \sum_{b, b} F_{b b^{\prime}}\left(b-b_{0}\right)\left(b^{\prime}-b_{0}\right)+\sum_{b, \theta} F_{b \theta}\left(b-b_{0}\right)\left(\theta-\theta_{0}\right)+\sum_{\theta, \theta} F_{\theta \theta^{\prime}}\left(\theta-\theta_{0}\right)\left(\theta^{\prime}-\theta_{0}^{\prime}\right) \\
& \sum_{b, \phi}\left(b-b_{0}\right)\left(V \cos \phi+V_{2} \cos 2 \phi+V_{3} \cos 3 \phi\right)+ \\
& \sum_{\theta, \phi}\left(\theta-\theta_{0}\right)\left(V \cos \phi+V_{2} \cos 2 \phi+V_{3} \cos 3 \phi\right)+\sum_{b, \theta, \phi} F_{b \theta \phi}\left(b-b_{0}\right)\left(\theta-\theta_{0}\right) \cos \phi+ \\
& \sum_{i, j} \frac{q_{i} q_{j}}{r_{i j}}+\sum_{i, j} \varepsilon_{i, j}\left[\frac{A_{i j}}{r_{i j}^{9}}-\frac{B_{i j}}{r_{i j}^{6}}\right]
\end{aligned}
$$

Here the first four terms describe bond stretching, bending, torsion and dihedral interactions, known as valence terms. Terms 5-11 are cross terms representing the coupling between different degrees of freedom of bond deformation. Term 12 is the Coulombic interaction between the charged atoms and term 13 gives the van der Waals interaction using the 9-6 Lennard-Jones potential. Coulombic forces were calculated using Ewald summation method while van der Waals interactions were summed by atom based truncation

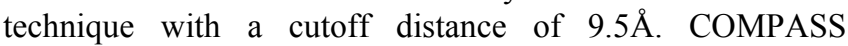
forcefield has been parameterized for a variety of organic chemical groups and any system containing any of these groups in any combination can be handled. Also, a number of metals have been parameterized, including copper in the current work. It should be pointed out that only van der Waals forces are counted for copper-copper and copper-epoxy interactions since there is no covalent bond across the interface.

Methodology of building crosslinked network structure for epoxy molding compound

The algorithm of building crosslinked polymer structure is based on close contact calculation, i.e., covalent bonds form between opposite reactive sites provided they are within predefined close contact. The process will be described below in full detail

Step 1: Monomer structures for BPA and tri/tetrafunctionality EPN1180 were prepared, with epoxy rings being opened and hydrogens being transferred form hydroxyl groups to epoxy oxygens. A number of monomers were then mixed randomly into a cubic cell with $3 \mathrm{D}$ periodic boundary condition using the Amorphous Cell module of Material Studio software. The ratio between the number of tri/tetrafunctionality EPN1180 and BPA is 8:12:36, assuring the amount of opposite reactive sites equal and the number averaged functionality of epoxy 3.6.

Step 2: The physical mixture of monomers aforementioned was then subjected to a iterative crosslinking procedure. Within each loop the mixture experienced an optimization-MD simulation-optimization-crosslinking process. The MD simulation ran under canonical (NVT) ensemble at $298 \mathrm{~K}$ for 500 steps with timestep of 0.5 femtosecond. It moved molecules and atoms around in the cell and allowed opposite reactive sites to get closer to each other. The distances between opposite reactive sites were calculated and if within a prescribed cutoff radius, covalent 
bonds formed between them. The structure was then optimized by energy minimization for 500 steps and the loop reran until not a single bond forms within one loop. All unreacted reactive sites were then saturated by hydrogen atoms. The conversion, defined as the fraction of either type of reactive site that has been reacted, of the current crosslinked model is $81.94 \%$.

Step 3: The final 3D crosslinked network structure was optimized by energy minimization to remove some unnatural bond stretching. Thereafter, the system was subjected to a NVT MD simulation at $600 \mathrm{~K}$ for $1 \mathrm{~ns}$ and a series of NVT runs from $600 \mathrm{~K}$ to $300 \mathrm{~K}, 10 \mathrm{ps}$ for every $10 \mathrm{~K}$, in order to remove construction history from the system, since the charges of reactive sites and cross-links are introduced artificially during the crosslinking process. Another MD simulation under Microcanonical (NPT) ensemble at $298 \mathrm{~K}$ and atmospheric pressure then conducted to get the structure equilibrated. Anderson and Berendson methods were utilized for thermostat and barostat in all MD simulations. The system density was finally equilibrated to $1.172 \mathrm{~g} / \mathrm{cm}^{3}$.

A snapshot of the final crosslinked structure for the epoxy molding compound is shown in Fig. 4. We note that the crosslinking procedure stated above is not intended to simulate the actual curing process. It meant to be an efficient way to arrive at the crosslinked network structure after curing with relatively high conversion. An important feature of the model is that links between reactive sites not only form within the cell but also across the boundaries due to periodicity. Thus a network extending to infinity is obtained.

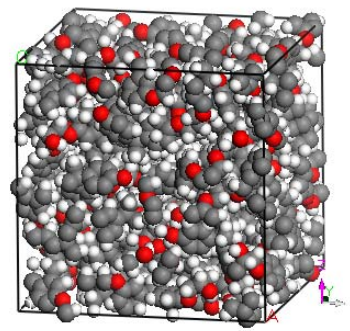

Figure 4. Crosslinked structure built for the epoxy molding compound

Production run for material properties of EMC

Once the equilibration process was completed, the EMC model was prepared for subsequent investigation of material properties. Molecular dynamics/mechanics simulations were conducted to study glass transition temperature, coefficient of thermal expansion and mechanical moduli, which are critical for the performance of epoxy resin as adhesive in electronic packaging.

\section{A. Temperature dependence of density and specific volume}

To study the temperature dependence of various thermodynamic quantities, an annealing molecular dynamics simulation was performed. The equilibrated model was first heated up to $500 \mathrm{~K}$ by a $1 \mathrm{~ns}$ NPT simulation and then cooled down to $300 \mathrm{~K}$ at a rate of $10 \mathrm{~K} / 100 \mathrm{ps}$ by a series of NPT runs under atmospheric pressure adjusted by Berendson barostat technique. Starting from the last three frames of the equilibration trajectory as initial configurations, this annealing process ran for three times and resulted thermodynamic quantities were averaged to improve accuracy. The temperature dependence of the system density is plotted in Fig. 5. A steady increase of density with decreasing temperature is observed, as is expected. Also, a kink in the fitted density versus temperature slope is seen, which is usually identified as the point when polymer transits from glassy to rubbery state, i.e., the glass transition temperature $\left(T_{\mathrm{g}}\right)$. In our case $T_{\mathrm{g}}$ is $398 \mathrm{~K}$, in good agreement with the experimentally determined glass transition temperature which ranges from $369 \mathrm{~K}$ for dilatometric data to $396 \mathrm{~K}$ for DMA data [13], indicating the validity of our model and the methodology of determining $T_{\mathrm{g}}$. Simultaneously obtained is the temperature dependence of specific volume of the cell, shown in Fig. 6. A kink in specific volume versus temperature slope is observed at $400 \mathrm{~K}$, quite close to the glass transition point indicated by the temperature dependence of density.

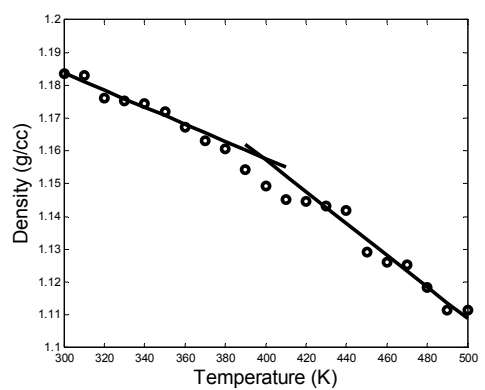

Figure 5. Temperature dependence of density

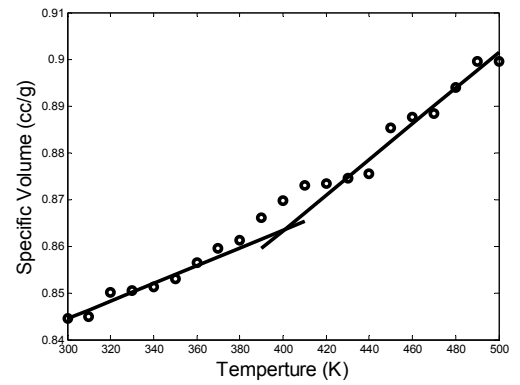

Figure 6. Temperature dependence of specific volume

The coefficient of thermal expansion (CTE) is another property of interest. It is of great important for thermo cycling life prediction in electronic packaging since the large mismatch of CTE of epoxy and substrate materials is a main source of interfacial delamination. Volumetric CET is defined as:

$$
\alpha=\frac{1}{V}\left(\frac{\partial V}{\partial T}\right)_{P}
$$

The fractional change of the system volume $(\mathrm{d} V / V)$ versus temperature is plotted in Fig. 7. A kink of slope indicative of $T_{\mathrm{g}}$ observed at $398 \mathrm{~K}$. According to Ea. 2, the fitted $\mathrm{d} V / V$ versus temperature slope in Fig. 7 is the volumetric CTE. In our case, it is calculated as $2.238 \times 10^{-4} \mathrm{~K}^{-1}$ for glassy state and $4.080 \times 10^{-4} \mathrm{~K}^{-1}$ for rubbery state, falling in the range of $1.5 \sim 3.5 \times 10^{-4} \mathrm{~K}^{-1}$ for glassy state and $4 \sim 8 \times 10^{-4} \mathrm{~K}^{-1}$ for rubbery state of typical thermosetting polymers [28]. Experimental data regarding volumetric CTE are available for a similar compound (also composed of EPN1180 and Bisphenol A) under external pressure of $10 \mathrm{MPa}$. It is around $1.93 \times 10^{-4} \mathrm{~K}^{-1}$ in glassy temperature range and $5.34 \times 10^{-4} \mathrm{~K}^{-1}$ in rubbery 
temperature range [29]. Therefore, our simulation results are in reasonable agreement with experimental values.

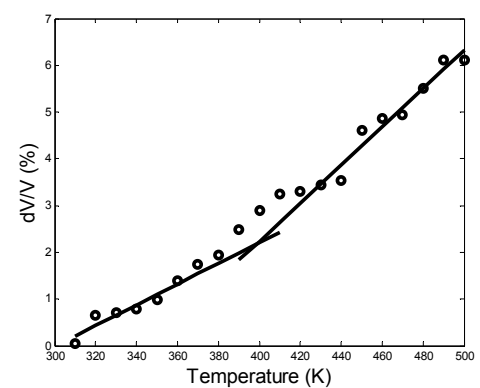

Figure 7. Dependence of fractional change of system volume on temperature

\section{B. Mechanical properties}

Elastic constants of the epoxy molding compound were calculated by a purely molecular statics method instead of molecular dynamics. A constant strain energy minimization procedure was applied to the final configuration of the previous equilibration MD simulation at $298 \mathrm{~K}$. The equilibrated crosslinked structure experienced an initial optimization followed by six deformation patterns, i.e., uniaxial tensions/compressions along three perpendicular directions and pure shears in $x y, y z$ and $z x$ planes. All these deformations were applied while keeping the remaining dimensions of the unit cell fixed. In each deformation pattern the structure was loaded for 10 steps and the maximum strain amplitude is 0.003 . The structure was re-minimized after each loading step and the internal stress tensor was obtained from the virial stress formulation:

$$
\boldsymbol{\sigma}=-\frac{1}{V_{0}}\left(\sum_{i=1}^{N} m_{i} \mathbf{v}_{i} \otimes \mathbf{v}_{i}+\sum_{i<j} \mathbf{r}_{i j} \otimes \mathbf{f}_{i j}\right)
$$

Upon getting the stress-strain response for one specific deformation pattern, components of the stiffness matrix were calculated as the second order derivate of potential energy with respect to strain or the first order derivate of stress with respect to strain, using a finite difference formula:

$$
\begin{gathered}
C_{i j k l}=\frac{1}{V} \frac{\partial^{2} U}{\partial \varepsilon_{i j} \partial \varepsilon_{k l}} \\
C_{i j k l}=\frac{\partial \sigma_{i j}}{\partial \varepsilon_{k l}}
\end{gathered}
$$

After this constant strain optimization is conducted for all the six deformation patterns, the complete stiffness matrix was obtained.

$\left[\begin{array}{cccccc}6.5491 & 3.3941 & 3.4986 & 0.0710 & 0.0763 & -0.1587 \\ 3.3941 & 7.6844 & 3.5593 & 0.5401 & 0.1039 & -0.2608 \\ 3.4986 & 3.5593 & 7.3233 & 0.0648 & 0.1285 & -0.0584 \\ 0.0710 & 0.5401 & 0.0648 & 1.1025 & 0.0745 & -0.0499 \\ 0.0763 & 0.1039 & 0.1285 & 0.0745 & 1.6489 & 0.0180 \\ -0.1587 & -0.2608 & -0.0584 & -0.0499 & 0.0180 & 1.4628\end{array}\right]$

It can be observed from the stiffness matrix that:

$$
C_{11} \approx C_{22} \approx C_{33}, C_{12} \approx C_{23} \approx C_{31}, C_{44} \approx C_{55} \approx C_{66}
$$

Although the rest components of the stiffness matrix are not rigorously zero, they are much smaller and can be considered as zero. Assuming the isotropicity of the material, as is the case for most thermosetting polymers, the Lame constants can be calculated as [30]:

$$
\mu=\frac{4 a-2 b+3 c}{33}, \lambda=\frac{2 a+c-15 \mu}{6}
$$

where

$a=C_{11}+C_{22}+C_{33}, b=C_{12}+C_{21}+C_{23}+C_{32}+C_{13}+C_{31}, c=C_{44}+C_{55}+C_{66}$

Once the Lame constants are obtained, mechanical properties including bulking modulus $(K)$, Young's modulus $(E)$, shear modulus $(G)$ and Poisson's ration $(v)$ can be derived as:

$$
\begin{aligned}
& E=\mu \frac{3 \lambda+2 \mu}{\lambda+\mu}, G=\mu \\
& K=\lambda+\frac{2}{3} \mu, v=\frac{\lambda}{2(\lambda+\mu)}
\end{aligned}
$$

The calculated elastic constants for the epoxy molding compound are summarized in table 1 .

Table 1. Mechanical properties of Epoxy Molding Compound from molecular statics simulation and experiment (for a similar compound)

\begin{tabular}{c|c|c}
\hline $\begin{array}{c}\text { Mechanical } \\
\text { properties }\end{array}$ & $\begin{array}{c}\text { Molecular } \\
\text { statics simulation } \\
\text { results }\end{array}$ & $\begin{array}{c}\text { Experimental } \\
\text { results }\end{array}$ \\
\hline $\begin{array}{c}\text { Bulk modulus } \\
\text { (GPa) }\end{array}$ & 5.313 & 5.220 \\
\hline $\begin{array}{c}\text { Young's } \\
\text { modulus (GPa) }\end{array}$ & 3.861 & 2.360 \\
\hline $\begin{array}{c}\text { Shear modulus } \\
\text { (GPa) }\end{array}$ & 1.405 & 0.830 \\
\hline Poisson's ratio & 0.377 & - \\
\hline
\end{tabular}

Since the above mechanical properties were calculated using purely static method, they should be understood as mechanical properties at $0 \mathrm{~K}$. Experimental data regarding the mechanical property of a similar compound (also composed of EPN1180 and Bisphenol A) at $50^{\circ} \mathrm{C}$ are available, as is summarized in the third column of table 1. Although these two sets of data cannot be compared directly, they are in reasonable agreement with each other. The simulated Poisson' ration falls into the range of $0.30 \sim 0.46$ for typical glassy state thermosetting polymers. Therefore, we conclude that the above mentioned molecular static simulation reproduce accurately mechanical properties for the epoxy molding compound.

\section{EMC/copper interfacial adhesion and separation A. interfacial adhesion energy}

To build an interface model, the epoxy molding compound needs to be attached to a copper substrate. To this end, constitutive monomers (EPN1180s and BPAs) were first packed into a confined layer (Fig. 8 (a)) in which the distribution of monomers along the perpendicular direction was limited. This layer of monomer mixture was then placed over a copper substrate with (111) free surface (Fig. 8 (b)). After that, the same crosslinking methodology as is used for bulk polymer model was applied to get monomers crosslinked to a 2D infinitely extended network with conversion of 
$84.3 \%$. The resulted epoxy molding compound/copper interface model (Fig. 8 (c)) has in-plane rather than 3D periodicity.

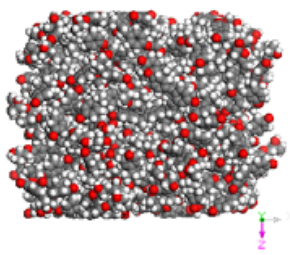

(a)

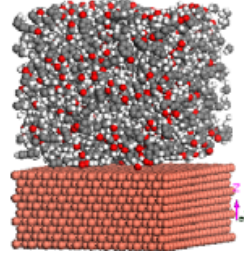

(b)

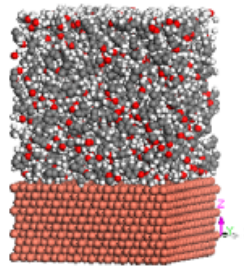

(c)

Figure 8. (a) layer of monomer mixture; (b) monomer mixture/copper system; (c) 2D polymer network/copper system

The interface model contained some unnaturally stretched bonds, as is the case in bulk EMC model construction. Energy minimization was carried out to removed these unnatural bond stretching and optimize the atomistic configuration. Once the optimized interface structure is obtained, the interfacial interaction energy between the two components (epoxy and copper) can be calculated as:

$$
\Delta E=E_{\text {total }}-\left(E_{C u}+E_{M C}\right)
$$

$E_{\text {total }}$ is the single point potential energy of the whole interface system. $E_{\mathrm{Cu}}$ and $E_{\mathrm{MC}}$ are the single point potential energy of the copper and polymer layers of the same interface system, respectively. The interfacial interaction energy of the current model is $-6729.862 \mathrm{~kJ} / \mathrm{mol}$. The area density of the interfacial interaction energy is defined as:

$$
\gamma=\Delta E / A
$$

For the current model this quantity is calculated as $0.573 \mathrm{~J} / \mathrm{m}^{2}$. Directly relevant proof of this results, either experimental or computational, is not available. However, work of adhesion calculated form MD simulation for the interface between alumina and a different type of epoxy resin (Phosphated epoxy cured by tetramethylol glycoluril) by Irene Yarovsky and Evan Evans [10] is available and the value is $0.67 \mathrm{~J} / \mathrm{m}^{2}$, which is consistent to our result..

\section{B. Traction-displacement law of the interface}

To extract the traction-displacement law for opening mode separation, the optimized EMC/copper interface model in part A was subjected to a molecular statics simulation of uni-axial tension along the direction normal to the interface. During the uni-axial tension simulation, the very top layer of the epoxy resin was fixed, and the bottom layer of the copper substrate is displaced along the $-z$ direction. After each displacement increment, energy minimization is conducted to relax the system. The Conjugate Gradient method with Fletcher-Reeves algorithm was selected to do the minimization and the convergence level was set to $0.5 \mathrm{kcal} /(\mathrm{mol} \cdot \AA)$. The total $\mathrm{z}$ direction atomic forces on all copper atoms were summed up after the energy minimization. The total force divided by the interface area gives the traction across the interface. These steps were repeated many displacement increments with the displacement of $0.25 \AA$ for the first four increments and $1 \AA$ for all the rest increments. The copper substrate was displaced for $100 \AA$ in total at the end of the simulated tensile test. Fig. 9 (a) demonstrates atomic configurations of the EMC/copper system at different loading steps. The obtained tractiondisplacement relationship is shown in Fig. 9 (b), with the individual snapshots shown in Fig. 9 (a) being labeled.

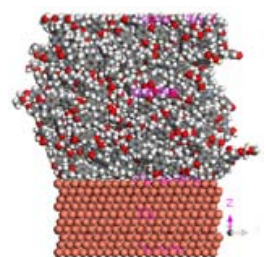

(1)

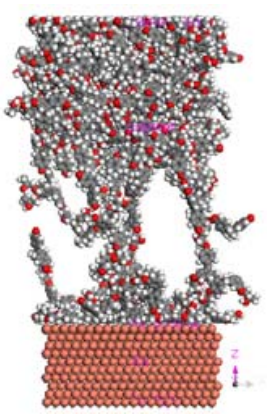

(4)

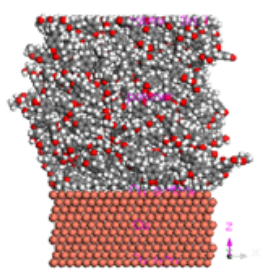

(2)

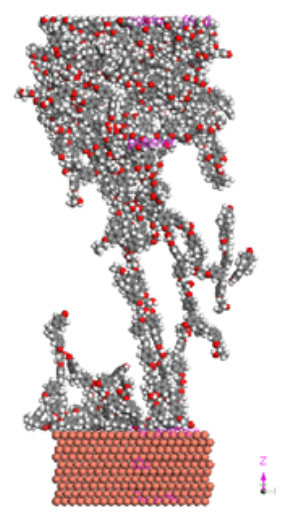

(5)

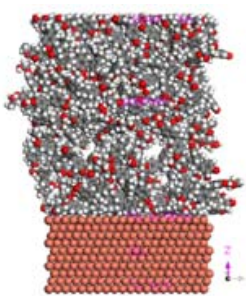

(3)

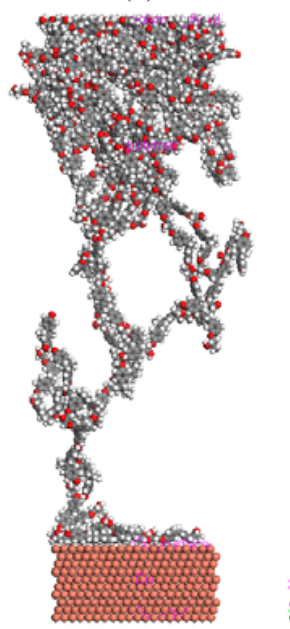

(6) (a)

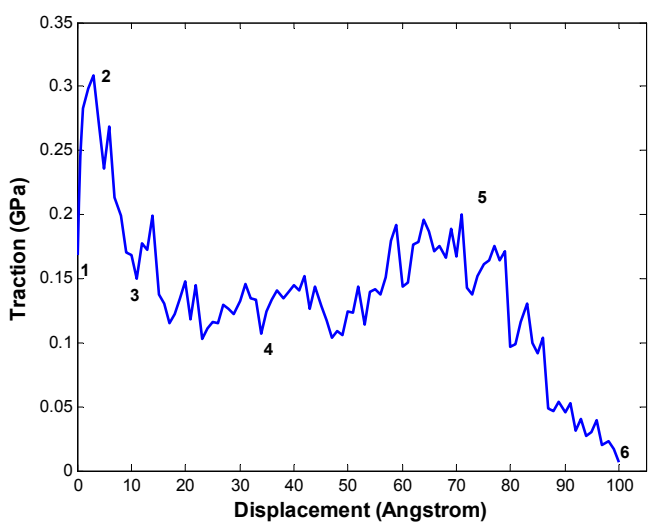

(b)

Figure 9. Opening mode separation of EMC/copper interface: (a) Atomic configurations at different loading steps. (b) Tractiondisplacement relationship 
Frame (1) in Fig. 9 (a) represents the initial configuration of the simulation. As the copper layer was translated downwards, the polymer underwent tensile deformation and the traction increased linearly to a peak value of $0.31 \mathrm{GPa}$ at about $3 \AA$ displacement (frame (2)), which corresponds to an average strain of $6.7 \%$ in the epoxy. Further displacement of the copper substrate caused sharp drop of traction and void initiation within epoxy resin (frame (3)). The voids grew in size and several chain segments of the polymer network became highly stretched (frame (4) and (5)) as the loading proceeded. At the end of the simulation (frame (6)), except for a few dangling monomers or oligomers remained attached to the copper free surface, the rest of the epoxy resin was completely separated from the copper substrate, and the traction dropped to almost zero. Viewing snapshots of the atomic configuration at different displacement steps, we can observe that damage first occurred within the polymer as voids caused by the breakage of inter-chain non-bonded interactions (Coulombic and van der Waals forces), rather than along the interface. However, as the load increases, the two phases started to separate, i.e., failure transited from cohesive to adhesive mode. Upon getting the tractiondisplacement relationship, the work of separation can be calculated as the area under the curve. Also can be extracted is the peak traction. The simulation results for these two quantities are listed in table 2.

Table 2. Work of separation and peak traction of the EMC/copper interface system

\begin{tabular}{c|c}
\hline $\begin{array}{c}\text { Work of separation } \\
\left(\mathrm{J} / \mathrm{m}^{2}\right)\end{array}$ & $\begin{array}{c}\text { Peak traction } \\
(\mathrm{GPa})\end{array}$ \\
\hline 1.372 & 0.3083 \\
\hline
\end{tabular}

\section{Conclusions}

The current article presents a molecular dynamics/statics study of a novel epoxy molding compound and its interface with copper substrate. A multi-step cross-linking algorithm based on an iterative MD simulation/energy minimization and close contact calculation was developed to build an atomistic model for the epoxy molding compound reflecting its network nature. The method is able to efficiently reach the post-cured structure of generic thermosets with relatively high conversion. Investigation of material properties, including glass transition temperature, volumetric coefficient of thermal expansion and elastic constants, based on the atomistic model was conducted and gave results in reasonably good agreement with experimental measurements. Performance of the interface between EMC and copper, in terms of interfacial adhesion energy and traction-displacement law, was also examined. The work of separation and peak traction were extracted from the traction-displacement law. These two quantities could be used in cohesive zone finite element simulation as key parameters to model interfacial delamination at the continuum scale, thus facilitate a hierarchical multi-scale simulation frame.

\section{Acknowledgments}

We thank the European Commission for partial funding of this work under project Nanointerface (NMP-2008-214371). Dr. Maria Entrialgo-Castano in Accelrys Inc. and Dr. Ole Hölck in Fraunhofer IZM, Germany are gratefully acknowledged for their insightful discussions on this topic.

\section{References}

1. Materials Studio Overview. Available from: http://accelrys.com/products/materials-studio/.

2. Bermejo, J.S. and C.M. Ugarte, Influence of CrossLinking Density on the Glass Transition and Structure of Chemically Cross-Linked PVA: A Molecular Dynamics Study. Macromolecular Theory and Simulations, 2009. 18(6): p. 317-327.

3. Doherty, D.C., et al., Polymerization molecular dynamics simulations. I. Cross-linked atomistic models for poly(methacrylate) networks. Computational and Theoretical Polymer Science, 1998. 8(1-2): p. 169-178.

4. Gou, J.H., et al., Computational analysis of effect of single-walled carbon nanotube rope on molecular interaction and load transfer of nanocomposites. Composites Part B-Engineering, 2005. 36(6-7): p. 524533.

5. Gou, J.H., et al., Computational and experimental study of interfacial bonding of single-walled nanotube reinforced composites. Computational Materials Science, 2004. 31(3-4): p. 225-236.

6. Komarov, P.V., et al., Highly cross-linked epoxy resins: an atomistic molecular dynamics simulation combined with a mapping/reverse mapping procedure. Macromolecules, 2007. 40(22): p. 8104-8113.

7. Varshney, V., et al., A molecular dynamics study of epoxy-based networks: Cross-linking procedure and prediction of molecular and material properties. Macromolecules, 2008. 41(18): p. 6837-6842.

8. $\mathrm{Wu}, \mathrm{C} . \mathrm{F}$. and W.J. Xu, Atomistic molecular modelling of crosslinked epoxy resin. Polymer, 2006. 47(16): p. 6004-6009.

9. Yang, W., et al., Molecular dynamics simulation of the formation of polymer networks. Macromolecular Theory and Simulations, 2007. 16(5): p. 548-556.

10. Yarovsky, I. and E. Evans, Computer simulation of structure and properties of crosslinked polymers: application to epoxy resins. Polymer, 2002. 43(3): p. 963-969.

11. Fan, H.B., et al., Investigation of moisture diffusion in electronic packages by molecular dynamics simulation. Journal of Adhesion Science and Technology, 2006. 20(16): p. 1937-1947.

12. Fan, H.B. and M.M.F. Yuen, Material properties of the cross-linked epoxy resin compound predicted by molecular dynamics simulation. Polymer, 2007. 48(7): p. 2174-2178.

13. Liao, N.B., et al., Investigation on Thermal Properties of Crosslinked Epoxy Resin by MD Simulation. Eurosime 2009: Thermal, Mechanical and Multi-Physics Simulation and Experiments in Micro-Electronics and Micro-Systems, 2009: p. 546-549. 
14. Mijovic, J. and H. Zhang, Molecular dynamics simulation study of motions and interactions of water in a polymer network. Journal of Physical Chemistry B, 2004. 108(8): p. 2557-2563.

15. Tack, J.L. and D.M. Ford, Thermodynamic and mechanical properties of epoxy resin DGEBF crosslinked with DETDA by molecular dynamics. Journal of Molecular Graphics \& Modelling, 2008. 26(8): p. 1269-1275.

16. $\mathrm{Yu}, \mathrm{S} ., \mathrm{S}$. Yang, and M. Cho, Multi-scale modeling of cross-linked epoxy nanocomposites. Polymer, 2009. 50(3): p. 945-952.

17. Lin, Y.C., Investigation of the moisture-desorption characteristics of epoxy resin. Journal of Polymer Research, 2006. 13(5): p. 369-374.

18. Liang, Z.Y., et al., Investigation of molecular interactions between $(10,10)$ single-walled nanotube and Epon 862 resin/DETDA curing agent molecules. Materials Science and Engineering a-Structural Materials Properties Microstructure and Processing, 2004. 365(1-2): p. 228-234.

19. Fan, H.B., et al., Molecular dynamics simulation of thermal cycling test in electronic packaging. Journal of Electronic Packaging, 2007. 129(1): p. 35-40.

20. Wong, C.K.Y., H.B. Fan, and M.M.F. Yuen, Interfacial adhesion study for SAM induced covalent bonded copper-EMC interface by molecular dynamics simulation. Ieee Transactions on Components and Packaging Technologies, 2008. 31(2): p. 297-308.

21. Fan, H.B., C.K.Y. Wong, and M.M.F. Yuen, A Multiscale Interfacial Delamination Model of Cu-SAM-Epoxy Systems. 2008 International Conference on Electronic Packaging Technology \& High Density Packaging, Vols 1 and 2, 2008: p. 285-289.

22. Fan, H.B., C.K.Y. Wong, and M.M.F. Yuen, $A$ Multiscale Method to Predict Delamination in $\mathrm{Cu}$ Epoxy Systems in Electronic Packages. 2009 Ieee 59th Electronic Components and Technology Conference, Vols 1-4, 2009: p. 246-250.

23. Adnan, A. and C.T. Sun, Effect of adhesive thickness on joint strength: A molecular dynamics perspective. Journal of Adhesion, 2008. 84(5): p. 401-420.

24. Awasthi, A.P., D.C. Lagoudas, and D.C. Hammerand, Modeling of graphene-polymer interfacial mechanical behavior using molecular dynamics. Modelling and Simulation in Materials Science and Engineering, 2009. 17(1): p. -.

25. Kulmi, U. and S. Basu, A molecular dynamics study of the failure modes of a glassy polymer confined between rigid walls. Modelling and Simulation in Materials Science and Engineering, 2006. 14(6): p. 1071-1093.

26. Jansen, K.M.B., Structure of Delft Molding compound. (private communication).

27. Sun, H., COMPASS: An ab initio force-field optimized for condensed-phase applications - Overview with details on alkane and benzene compounds. Journal of Physical Chemistry B, 1998. 102(38): p. 7338-7364.
28. Jean-Pierre Pascault, H.S., Jacques Verdu, Roberto J. J. Williams, Thermosetting Polymers. 2002, New York: Marcel.

29. Jansen, K.M.B., (private communication).

30. Suter, U.W. and B.E. Eichinger, Estimating elastic constants by averaging over simulated structures. Polymer, 2002. 43(2): p. 575-582. 